\title{
Is freezing an adaptive reaction to threat? Evidence from heart rate reactivity to emotional pictures in victims of war and torture
}

\author{
HANNAH ADENAUER,,${ }^{a}$ CLAUDIA CATANI, ${ }^{\mathrm{b}}$ JULIAN KEIL, ${ }^{\mathrm{a}}$ HANNAH AICHINGER,,${ }^{\mathrm{a}}$ AND. \\ FRANK NEUNER ${ }^{b}$ \\ a'Department of Psychology, University of Konstanz, Konstanz, Germany \\ ${ }^{b}$ Department of Psychology, Bielefeld University, Bielefeld, Germany
}

\begin{abstract}
The influence of past traumatic experiences on the defense cascade in response to affective pictures was examined in survivors of war and torture. Trauma-exposed refugees with and without Posttraumatic Stress Disorder (PTSD) as well as healthy individuals viewed 75 pictures that varied in emotional content. Heart rate (HR) was recorded during the flickering stimulation of affective pictures in the context of a steady-state experiment. Whereas healthy controls showed the typical orienting response to aversive stimuli, PTSD patients reacted with an almost immediate increase in HR toward unpleasant pictures. Trauma-exposed participants without PTSD showed an indiscriminate orienting response regardless of picture category. The present findings argue for a faster flight/fight response to threatening cues in PTSD. In contrast, trauma-exposed controls seem to exhibit a state of permanent alertness toward a wide range of stimuli.
\end{abstract}

Descriptors: Anxiety, Psychopathological, Heart rate

The exposure to life-threatening traumatic events can bring about a variety of long-lasting changes in human experience and behavior. Typical consequences interfering with functioning, including repetitive intrusive recollections of the trauma and persistent avoidance of trauma reminders, have been integrated into the diagnosis of posttraumatic stress disorder (PTSD). Physiological symptoms are often the most prominent characteristics of PTSD and include symptoms of chronic hyperarousal. This is apparent in increased irritability and sleeping difficulties. Furthermore, PTSD patients react to reminders of the traumatic event with an increased physiological reactivity. Intrusive recollections of the event are often accompanied by high arousal (Carson et al., 2007; Elbert, Rockstroh, Kolassa, Schauer, \& Neuner, 2006).

We are grateful to all participants who took part in the study. The authors thank the outpatient clinic staff, especially Martina Ruf and Maggie Schauer, for clinical support, Heike Riedke for logistical support, Ursula Lommen for helping with physiological data acquisition, Stephan Moratti and Christian Wienbruch for technical advice, and Katy Robjant for editing and Sonja Haas and Steivan Pinösch for entry and ad ministration of data. The corresponding author has full access to all of the data in the study and takes responsibility for the integrity of the data and the accuracy of the data analysis. The research was supported by the Deutsche Forschungsgemeinschaft (DFG) and the European Refugee Fund (ERF). Authors declare they have no conflict of interest.

Address reprint requests to: Hannah Adenauer, University of Konstanz, 78457 Konstanz, Germany. E-mail: Hannah.Adenauer@ uni-konstanz.de
The chronic hyperarousal and the excessive reactivity to trauma reminders indicate a dysfunctional regulation of the physiological stress system in PTSD patients. Abnormalities in the physiological system have been confirmed in psychophysiological research (Buckley \& Kaloupek, 2001). Several studies in PTSD have shown an elevated reactivity to auditory and visual cues associated with the trauma across a variety of physiological measures, including heart rate (HR), blood pressure (BP), skin conductance (SC), and facial electromyograms (EMGs; Orr \& Roth, 2000). Moreover, studies have shown that high resting heart rate in the first days after the traumatic event predicts chronic PTSD (Bryant, 2006; Shalev et al., 1998).

Some authors have interpreted the elevated physiological reactivity to trauma reminders as an indicator of an excessive stress reaction of trauma survivors. Within the classical stress-response model, the immediate increase of arousal triggered by threat is interpreted as a normal reaction, priming the organism for a rapid fight or flight response. In this context, the enhanced physiological response of the PTSD patients might be interpreted as an adaptive reaction, preparing the trauma survivors for survival in a life-threatening context (Silove, Steel, McGorry, \& Mohan, 1998).

However, animal research has shown that an immediate increase of the sympathetic nervous system following a threat cue does not sufficiently account for the defensive reaction of animals (Fanselow, 1994; Timberlake, 1993). Rather, reflex reactivity in defense is organized sequentially, reflecting the proximity or imminence of a threat (Bradley, Codispoti, Cuthbert, \& Lang, 
2001). In particular, the fight or flight response is preceded by a short period of freezing behavior, characterized by orienting and information gathering (Bracha, 2004; Gray, 1987). Using finegraded measurement of physiological reactions to emotional pictures, Lang, Davis, and Ohman (2000) suggested that defensive responding in humans is similarly staged, a phenomenon known as "defense cascade." Contrary to the classical stress response model and in line with the defense cascade perspective, several studies have found that the initial reaction to aversive stimuli is characterized by a decrease rather than an increase of heart rate and is accompanied by an inhibition of the startle response (Bradley, Codispoti, Cuthbert, et al., 2001; Lang et al., 2000). A few seconds after the onset of a threatening stimulus, the direction of the physiological response reverses toward cardiac acceleration and an increase of the startle reflex. The transient pause before the action mobilization is associated with heightened sensory perception and processing of contextual details and has been interpreted as the human counterpart of the freezing state in animals (Graham, 1979; Sokolov, 1963). Therefore, in the present article, we use the term "freezing" to refer to the very early orienting response toward aversive stimulation. In contrast, "freezing" in this sense does not refer to the stage "tonic immobility" (Bracha, 2004) that is associated with later processing stages of threat-related cues.

Although a few studies have applied the concept of orienting response to research in PTSD (Elsesser, Sartory, \& Tackenberg, 2004), we have found no study with PTSD patients that analyzed heart rate data following affective stimulation at sufficient temporal resolution to quantify the initial heart rate deceleration in detail. As the decrease of arousalduring the orienting response is minimal and transient in comparison to the following fight/fligh state, it is difficult to detect and therefore may have been overlooked in past research. As alterations of the defense cascade are a central feature of PTSD, it is essential to understand these processes in detail. Thus, the aim of the present study was to examine whether repeated traumatic experiences in the context of war and torture are correlated with alterations in the defense cascade, with a particular focus on the orienting response. As heart rate stands out as one of the most reliable correlates of PTSD across all types of psychophysiological PTSD research (Pole, 2007) and as an increase in heart rate is a sensitive marker of PTSD-related reactivity to trauma-related stimuli (Blanchard et al., 1996; Miller \& Litz, 2004), we chose heart rate as the dependent measure for autonomic arousal change. As we aimed at finding evidence for alterations of the defence cascade, we used a standard set of emotional (pleasant, unpleasant, and neutral) pictures that has been shown to trigger defensive reactions before (Miller \& Litz, 2004).

Although the number of traumatic event types is the main predictor for the development of chronic PTSD in war survivors (Neuner et al., 2004), human beings can be astonishingly resilient after traumatic experiences. Many survivors of war and torture do not fulfill the diagnosis of PTSD or recover spontaneously. To account for this, we compared the response pattern of three groups: survivors with a high number of war and torture experiences with a diagnosis of PTSD (PTSD group) and without PTSD (resilient group) and healthy controls with no or almost no past traumatic experience (unexposed group). We expected to replicate the HR response pattern that has often been found in healthy participants. That is, unpleasant pictures prompt the greatest orienting response (indicated by an initial deceleration) followed by pleasant and by neutral pictures, respectively (Brad- ley, Codispoti, Cuthbert, et al., 2001). In addition, we assumed that PTSD patients would respond with an almost immediate elevation in HR response toward aversive pictures mirroring a high threat state (approaching circa-strike of the predator). Similar results have been obtained with individuals with specific phobia (Hamm, Cuthbert, Globisch, \& Vaitl, 1997). For the resilient participants we hypothesized a response pattern falling in between the two other groups, mirroring a cumulative or doseresponse-like relationship of traumatic events and damage to mental health (Neuner et al., 2004).

\section{Methods}

\section{Participants}

Fifty-nine war and torture-exposed participants and 19 comparison individuals with no prior war and torture experiences, matched for ethnicity, participated in the study. Trauma-exposed participants were asylum seekers with a history of persecution, war, and torture who came for treatment or expert opinion to the Psychotrauma Research and Outpatient Clinic for Refugees, located at the Centre for Psychiatry, Reichenau, Germany. This trauma-exposed group was divided according to PTSD diagnosis. Thirty-nine (17 female, 22 male) participants fulfilled DSMIV criteria for current PTSD (PTSD group) and 20 ( 13 female, 7 male) survivors of war and torture experiences had not developed PTSD or had already recovered (resilient group). The 19 (11 female, 8 male) comparison participants were recruited by searching for migrants born in conflict regions with hardly any past traumatic experiences by announcements on campus bulletin boards (unexposed group).

PTSD patients were significantly older than the unexposed subjects and less educated when compared to the other two groups. There were no significant differences between unexposed and resilient participants with respect to age and education. Moreover, the three groups differed with respect to asylum status, with fewer PTSD patients having a safe asylum status compared to the other two groups.

All participants underwent an extensive standardized clinical interview administered by experienced psychologists and trained translators. The number of prior trauma experiences was assessed by means of the event checklist of the Clinician Administered PTSD Scale (CAPS; Blake et al., 1995) and the Vivo checklist of war, detention, and torture events (Vivo, 2006). Participants in the trauma-exposed group differed in their past experiences of war and torture events, while both groups showed an equally high prior trauma-load of CAPS events.

The CAPS was used for the diagnosis of PTSD and rating of PTSD symptoms (severity and frequency). Naturally, PTSD patients displayed the highest PTSD severity score, and the resilient participants reported more PTSD symptoms than the almost symptom free unexposed group. Current comorbid DSM-IVaxis one disorders were assessed with the MINI International Neuropsychiatric Interviews (M.I.N.I.; Sheehan et al., 1998). The following comorbid diagnoses were present in the PTSD sample: 28 depressive, 4 dysthymic, 2 alcohol abuse, and 1 alcohol dependence disorders. Within the resilient group there were 2 major depressive and 2 dysthymic disorders, and in the unexposed group 1 participant suffered from depressive and 2 participants from dysthymic comorbid disorders. None of the study participants fulfilled the criteria of current or past schizophrenic, paranoid, or other psychotic symptoms. 
Table 1. Demographic and Clinical Characteristics of the Three Subject Groups

\begin{tabular}{|c|c|c|c|c|c|}
\hline & & $\operatorname{PTSD}(n=39)$ & Resilient $(n=20)$ & Unexposed $(n=19)$ & $p$ \\
\hline \multicolumn{6}{|l|}{ Demographic data } \\
\hline Sex & & & & & 0.26 \\
\hline Female & $N(\%)$ & $17(43.6)$ & $13(65.0)$ & $11(57.9)$ & \\
\hline Male & $N(\%)$ & $22(56.4)$ & $7(35.0)$ & $8(42.1)$ & \\
\hline Age & $M(S D)$ & $34.05(9.15)$ & $29.35(9.06)$ & $27.32(9.43)$ & 0.02 \\
\hline Education (years) & $M(S D)$ & $8.26(3.31)$ & $11.85(1.87)$ & $12.37(1.21)$ & 0.001 \\
\hline Regions of origin & & & & & 0.06 \\
\hline Middle East & $N(\%)$ & $22(56.4)$ & $11(55.0)$ & $6(31.6)$ & \\
\hline The Balkans & $N(\%)$ & $5(12.8)$ & $5(25.0)$ & $10(52.6)$ & \\
\hline Africa & $N(\%)$ & $10(25.6)$ & $4(20.0)$ & $3(15.8)$ & \\
\hline Caucasus & $N(\%)$ & $2(5.1)$ & - & - & \\
\hline Asylum status (insecure) & $N(\%)$ & $34(87.2)$ & $5(25.0)$ & $1(5.3)$ & 0.001 \\
\hline \multicolumn{6}{|l|}{ Clinical data } \\
\hline \multicolumn{6}{|l|}{ Events } \\
\hline No. traumatic events & & & & & \\
\hline CAPS Event-Types & $M(S D)$ & $6.97(2.08)$ & $5.65(1.42)$ & $1.68(0.89)$ & 0.001 \\
\hline \multicolumn{6}{|l|}{ Vivo checklist } \\
\hline No. war \& torture types & $M(S D)$ & $10.74(5.61)$ & $3.95(5.56)$ & $0.42(1.02)$ & 0.001 \\
\hline Raped & $N(\%)$ & $14(35.9)$ & $2(10)$ & - & 0.003 \\
\hline \multicolumn{6}{|l|}{ Clinical symptoms } \\
\hline CAPS Score & $M(S D)$ & $79.79(18.51)$ & $16.25(21.67)$ & $3.68(9.01)$ & 0.001 \\
\hline SOMS-7 & $M(S D)$ & $27.05(12.31)$ & $9.05(10.54)$ & $3.84(5.73)$ & 0.001 \\
\hline HRSD & $M(S D)$ & $25.41(7.84)$ & $7.45(7.61)$ & $3.11(5.8)$ & 0.001 \\
\hline \multicolumn{6}{|l|}{ Vulnerability score } \\
\hline CAPS score/no. war \& torture & $M(S D)$ & $9.89(7.14)$ & $4.39(6.45)$ & $1.00(1.73)$ & 0.01 \\
\hline
\end{tabular}

Note: For continuous variables, ANOVAs were calculated; for dichotomous variables, chi-square tests were applied.

The Hamilton Rating Scale for Depression (HRSD; Williams, 1988) was administered to determine the severity of depressive symptoms, and the Screening for Somatoform Symptoms (SOMS-7; Rief, Hiller, \& Heuser, 1997) for the assessment of somatic problems revealed significant differences between the groups in reported depressive as well as psychosomatic symptoms. Equivalent to the PTSD severity, the most somatic and depressive symptoms were reported in the PTSD sample, followed by the resilient participants and the unexposed participants, respectively.

Psychoactive medications taken by the PTSD group were antidepressants $(n=11)$, hypnotics $(n=7)$, neuroleptics $(n=5)$, and anxiolytics $(n=2)$. The resilient group was significantly less medicated: antidepressants $(n=2)$, hypnotics $(n=1)$, and neuroleptics $(n=1)$. None of the participants in the unexposed group took psychoactive medication at the time of assessment.

Descriptive results as well as significant group differences in demographic and clinical variables are presented in Table 1.

\section{Stimuli and Presentation Procedure}

Based on normative pleasure and arousal ratings, 25 high-arousing unpleasant (e.g., mutilations, assaults, etc.), 25 pleasant (e.g., sports, children, etc.), and 25 neutral (e.g., neutral faces, household objects, etc.) pictures were chosen from the International Affective Picture System (IAPS; Lang, Bradley, \& Cuthbert, 2005). ${ }^{1}$ The three categories differed significantly from each other in IAPS normative valence ratings (pleasant: 7.4, neutral:

'The numbers of IAPS pictures were as follows: pleasant: 2190,2214 $2215,2383,2440,2480,2516,2840,2850,5130,5510,5740,7035,7175$, $7217,7491,7500,7590,7595,7700,8190,5830,5660,4607,2209$; neutral: 1722, 2030, 2058, 2165, 2216, 2311, 2340, 2345, 2352, 4599, 4608, $4641,4653,4660,5260,5700,8185,8200,8380,8496,7490,7130,5390$ 2570, 2410; unpleasant: 2120, 2900, 3181, 3301, 6190, 6212, 6250, 6312, $6540,6560,6831,6838,9040,9181,9400,9405,9415,9421,9433,9911$, $6821,3550,3530,2800,2053$.
4.9, unpleasant: 2.4). Arousal ratings did not differ for pleasant and unpleasant contents, but mean arousal levels for both emotional categories were significantly higher than for neutral contents (pleasant: 5.6, neutral: 2.9, unpleasant: 5.8.) Brightness, contrast, and color spectra of the stimuli were matched across picture categories.

As the study included the measurements of the steady-state visual evoked field (not reported here), the pictures were presented for the duration of $4 \mathrm{~s}$ in a flickering mode, at a $10-\mathrm{Hz}$ cycle, resulting in 40 on/off cycles. Pictures were shown on a white plastic screen in a pseudorandom order, with the restriction that no more than three pictures of the same affective category could occur in a row. The interstimulus interval varied randomly between 6 and $8 \mathrm{~s}$.

\section{Heart Rate Recording and Processing}

Two $\mathrm{Ag} / \mathrm{AgCl}$ electrodes positioned on the left and right inner forearms recorded heart rate activity. The usual procedure of taking heart rate recordings from the lower rib cage was considered inappropriately intimate for the multicultural and severely traumatized participants. Heart rate data were amplified with a Synamps (Neuroscan Laboratories, Sterling, VA) and digitized online at a rate of $678.17 \mathrm{~Hz}$

Heart rate changes following presentation of affective pictures were evaluated using an in-house algorithm written in MATLAB (Moratti, Keil, \& Stolarova, 2004). The algorithm estimated the heart rate change over $4 \mathrm{~s}$ of picture presentation in 250 -ms steps using a 1-s prestimulus baseline.

The HR data were scored according to the phasic model of HR change proposed by Hodes, Cook, and Lang (1985). That is, the minimum beats per minute (bpm) value during the first $2 \mathrm{~s}$ after stimulus onset was scored as the initial deceleration and the maximum value within the next $2 \mathrm{~s}$ was considered as the following acceleration. 


\section{Procedure}

The clinical interviews were carried out 1 week before HR recording in order to control for influences caused by the potential emotional priming by the diagnostic interview. The testing took place in a magnetically shielded chamber because magnetencephalographic (MEG) data were recorded as well (not reported here).

Upon arrival at the laboratory, the participants were provided with a full verbal and written explanation of the procedures and gave informed consent to participate. After HR recording, participants rated the 75 affective pictures for emotional valence and arousal using the Self-Assessment Manikin (SAM) self-report scale (Lang, Bradley, \& Cuthbert, 1999).

\section{Statistical Analysis}

Demographic and clinical variables were compared using repeated-measures analysis of variance (ANOVA) for continuous variables and the chi-square test for categorical variables. SAM pleasure and arousal ratings were evaluated using repeated-measures ANOVA with group as the between-subjects factor and emotional content (pleasant, neural, and unpleasant) as the within-subjects factor.

Heart rate data were examined for the two different phases (deceleration and acceleration) separately. We applied for each phase a repeated-measures ANOVA with emotional content (pleasant, neural, and unpleasant) as the within-subjects factor and group as the between-subjects factor. Where appropriate, the degrees of freedom were corrected in all ANOVAs using the Greenhouse and Geisser (1959) procedure to account for possible violations of the sphericity assumption. Fisher Least Significant Different (LSD) tests were used to investigate significant interaction effects. A linear regression model was applied to examine potential predictors of heart rate change in aversive picture processing.

\section{Results}

\section{SAM Ratings}

As expected, SAM pleasure ratings differed as a function of affective category, $F(2,114)=1053.70, p<.001$, with pleasant pictures rated as most pleasant followed by neutral pictures and unpleasant pictures rated as least pleasant across all participants.
Moreover, analyses of the valence ratings revealed a significant main effect of group, $F(2,57)=9.37, p<.001$. PTSD patients rated all pictures as less pleasant with respect to the two comparison groups (Fisher LSD $p<.01$ for all comparisons).

Arousal ratings for all participants also varied over affective categories, with unpleasant and pleasant pictures rated as more arousing than neutral pictures, $F(2,114)=200.51, \quad p<.001$ (Fisher LSD $p<.001$ for all comparisons). Arousal ratings also differed significantly between unpleasant and pleasant pictures (Fisher LSD $p<.001$ ). Moreover, SAM arousal ratings revealed a significant interaction effect of affective category by group, $F(4,114)=5.03, p<.001$. There was a trend that PTSD patients rated pleasant pictures as less arousing and unpleasant pictures as more arousing than the unexposed group (Fisher LSD $p>.05$ for both comparisons; Figure 1).

\section{Heart Rate Response}

We compared the prestimulus HR of our three groups. There was no significant difference in the averaged prestimulus $\mathrm{HR}$ level between the three participant groups, $F(2,75)=0.74, p>.05$. Regarding the whole stimulation interval of $4 \mathrm{~s}$, every group displayed a very distinct cardiac response pattern toward the three different picture categories. The mean heart rate change relative to prestimulus baseline for each group and for each category (pleasant, neutral, and unpleasant) is depicted in Figure 2.

Moreover, the cardiac response of the $4 \mathrm{~s}$ of picture presentation showed two distinct phases for all affective categories and all groups as detected by a main effect of phase, $F(1,75)=108.62$, $p<.001$. Characteristic of this biphasic model was an initial deceleration and a following acceleration (Fisher LSD $p<.001$ ).

Within the deceleration phase (in the first $2 \mathrm{~s}$ of picture presentation) there was a significant interaction of affective category by group, $F(4,150)=3.74, p<.01$. This difference was characterized by a very weak deceleration toward aversive stimuli in the PTSD group compared to a pronounced deceleration toward the aversive material in the two other groups (Fisher LSD $p<.01$ for the resilient and $p<.05$ for the unexposed group). A main effect of group revealed that the resilient sample showed a stronger initial deceleration, $F(2,75)=6.02, p<.005$, over all picture categories compared to the PTSD group (Fisher LDS $p<.001$ ). Intriguingly, these individuals did not display any differences in their HR response patterns between the affective categories (LSD

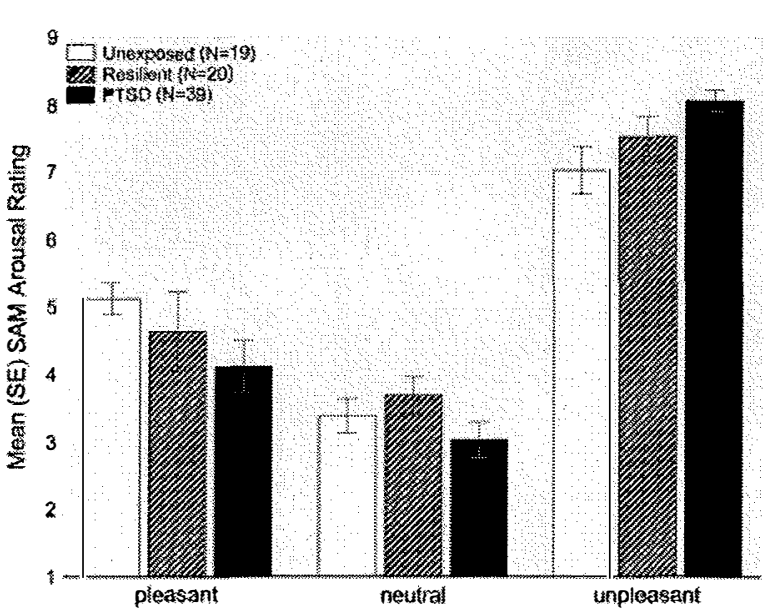

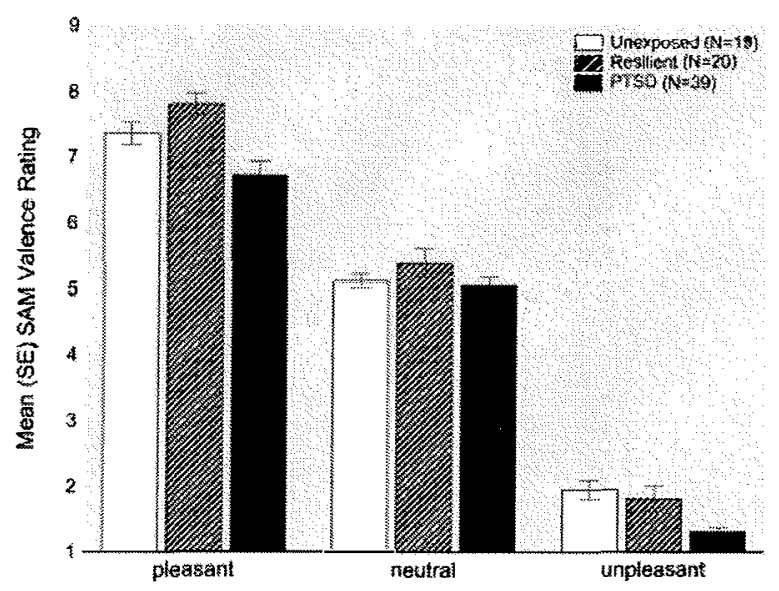

Figure 1. SAM valence (left) and SAM arousal (right) ratings of the three groups. 


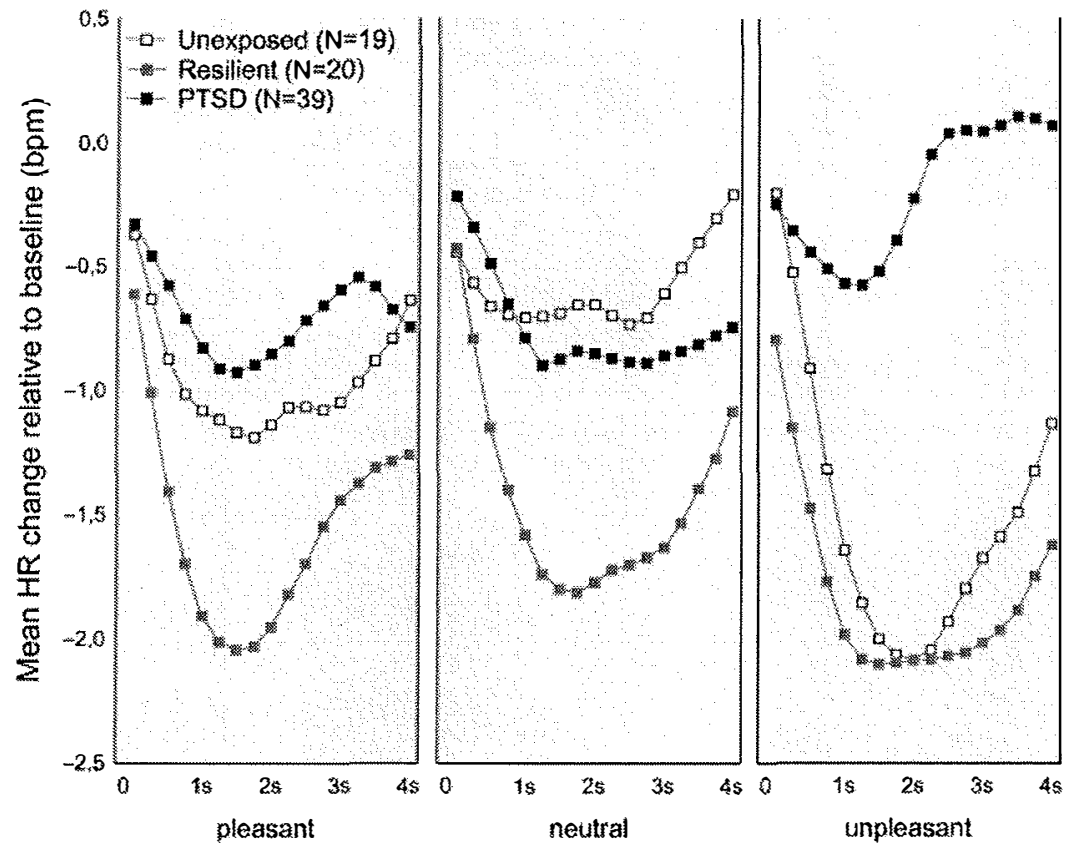

Figure 2. The mean heart rate (bpm) changes relative to prestimulus baseline across picture presentation ( $4 \mathrm{~s})$ for each group and for each category (pleasant, neutral, unpleasant).

$p>.2$ for all comparisons). Whether pleasant, neutral, or unpleasant pictures were shown, resilient individuals reacted with a pronounced cardiac deceleration. Finally, the unexposed group revealed the expected HR response (Bradley, Codispoti, Cuthbert, et al., 2001), characterized by an initial deceleration toward all pictures, that was most pronounced for the threatening picture content: main effect of affective category, $F(2,36)$ $=4.76, p<.01$ (Fisher LSD $p<.001$ for the comparison of neutral vs. unpleasant and $p>.05$ for the comparison pleasant vs. unpleasant).

Within the acceleration phase of picture presentation, a significant interaction of affective category and group, $F(4,150)=$

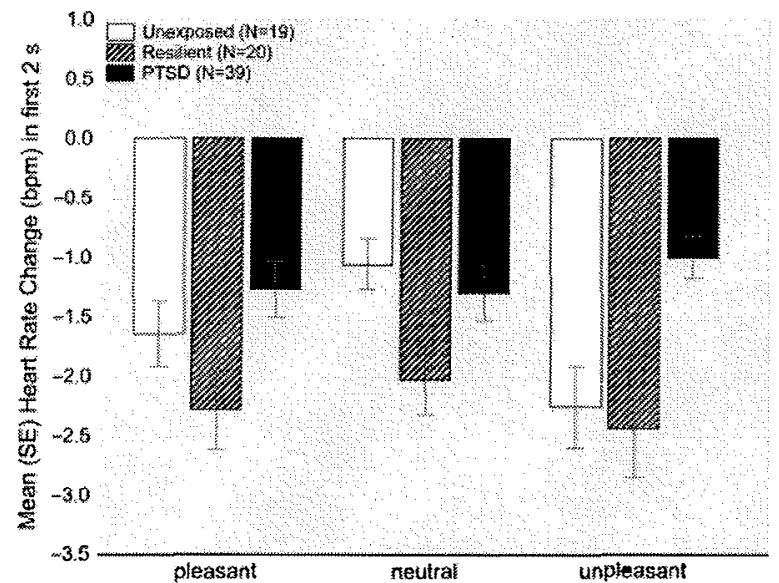

$5.22, p<.001$, revealed that the effect of a very distinct response pattern of the three groups was preserved. In the last $2 \mathrm{~s}$ of picture presentation a main effect of group, $F(2,75)=9.48, p<.001$, showed a more pronounced acceleration in the PTSD sample compared to the other two groups (LSD $p<.005$ for both comparisons). This strong acceleratory response in PTSD individuals was most distinct during the presentation of high arousing aversive images (Fisher LSD $p<.01$ in comparison to pleasant and $p<.001$ compared to neutral pictures). For both phases separately, HR changes relative to a prestimulus baseline for all groups and affective categories are depicted in Figure 3.

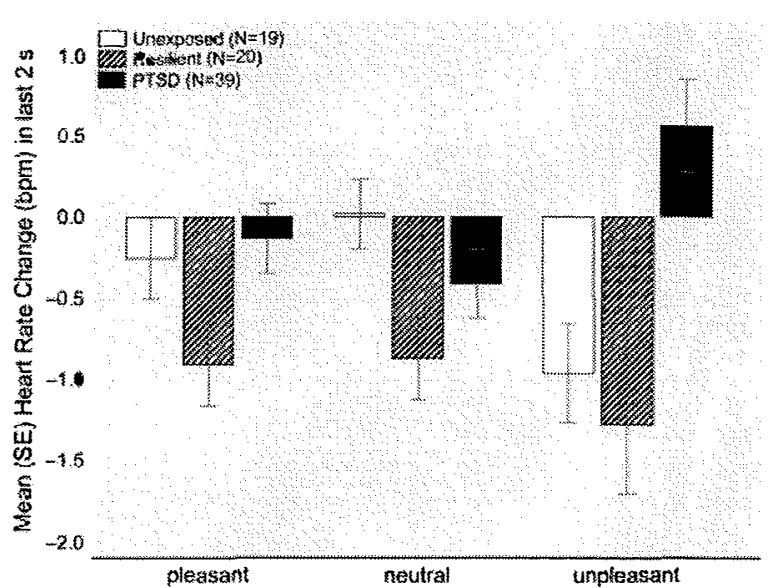

Figure 3. The mean heart rate (bpm) changes relative to prestimulus baseline for each group and for each category (pleasant, neutral, unpleasant) for the initial deceleration (left) and the following acceleration (right). 
Given the existing scientific evidence for distinct HR responses toward aversive cues between men and women (Bradley, Codispoti, Sabatinelli, \& Lang, 2001), we calculated an ANOVA with gender as an additional factor, both for the initial deceleration as well as the following acceleration of the HR response. Analyses showed that for both time intervals, gender did not have any significant effect. Any interaction yielded statistical significance: initial deceleration, $F(4,144)=2.14, p>.05$; acceleration, $F(4,144)=0.35, p>.05$. However, the main effect for group and the interaction between group and category remained statistically significant even if gender was included.

Prediction of Initial Heart Rate Changes toward Aversive Pictures To examine potential predictors of the heart rate change toward aversive images in the first two seconds after picture presentation, a linear regression model was calculated. Variables that were potentially related to the cardiac response toward aversive visual stimuli were included as the predictor variables. Because our main focus was the orienting response, the initial reaction served as the dependent variable. The "initial reaction" was defined as the minimum bpm value during the first $2 \mathrm{~s}$ after stimulus onset. The unexposed participants were excluded from the analysis because many individuals in this group reported zero or very few PTSD symptoms and few experiences of war and torture. Results of the analysis are presented in Table 2.

The main predictor of the initial $\mathrm{HR}$ response toward aversive images was the "vulnerability factor." This factor was created by the quotient of posttraumatic symptoms (CAPS sum score) and the number of past war and torture experiences. Accordingly, individuals with a low vulnerability factor were characterized by reporting few symptoms, despite having experienced many war and torture events.

The positive relation between vulnerability and the initial HR response reveals that vulnerable people show a relatively smaller HR deceleration within the first $2 \mathrm{~s}$. Neither age, depressive symptoms, asylum status, nor the experience of rape had a significant influence on the initial heart rate response toward aversive stimuli. Gender resulted as a further significant predictor, with women showing a comparatively stronger deceleration in this time interval.

Table 2. Linear Regression Model with the Initial Response toward Aversive Pictures as the Dependent Variable

\begin{tabular}{lcc}
\hline \hline Predictor & Zero-order correlation & $\beta$ \\
\hline Age & $0.30^{* *}$ & 0.14 \\
Gender (female) & $-0.29^{*}$ & $-0.36^{* *}$ \\
Vulnerability factor $^{\text {a }}$ & $0.39^{* *}$ & $0.41^{* *}$ \\
Raped (yes) & -0.18 & -0.09 \\
Depression $_{\text {Immigration status (safe) }}$ & $0.28^{*}$ & 0.02 \\
\hline \hline
\end{tabular}

Note: Zero-order correlation is represented by Spearman's rho for continuous predictor variables and point-biseriel correlation for dichotomous predictor variables; $\beta$ coefficients are standardized and results from a linear regression model of HR acceleration within the first $2 \mathrm{~s}$ of picture presentation; full model adjusted $R^{2}=.28$ for the PTSD and the resilient group.

avulnerability factor: CAPS-severity score divided by number of war and torture events.

${ }^{\mathrm{b}} \mathrm{HRSD}$ sum score.

${ }^{*} p<.05,{ }^{* *} p<.01$

\section{Discussion}

In agreement with previous research (Miller \& Litz, 2004), we found a cardiac acceleration in response to aversive pictures in patients with PTSD. HR acceleration following aversive stimuli was significantly greater in PTSD patients compared to the other two groups. Furthermore, in comparison to both the unexposed and the resilient groups, the orienting response to aversive pictures was almost absent in participants with PTSD. This shows that the heart rate response in the face of threat is not only stronger but also faster in those with a diagnosis of PTSD. Our results might indicate that in PTSD the defense cascade had been adjusted to allow for a rapid flight response to threatening cues without any further exploration of the stimulus. This finding is consistent with the observation that PTSD patients react to threatening cues and trauma reminders with a hypersensitive alarm system (Rauch et al., 2000). Furthermore, our findings might have implications for the interpretation of the attentional bias toward threat cues that has been documented in PTSD (Bryant \& Harvey, 1997; Buckley, Blanchard, \& Neill, 2000). Our findings suggest that, immediately after having identified the threatening quality of a stimulus, PTSD participants mobilize for action rather than exploring the stimulus further in a hypervigilant state.

Contrary to our assumptions, the resilient group did not show a reaction in between the unexposed group and the PTSD patients but reacted with a specific pattern. In this group, a pronounced indiscriminate orienting response was found for all classes of stimuli including the neutral pictures. These participants who did not develop or who had recovered from PTSD, despite a history of severe past traumatic events, remain in a permanent alert state, which is demonstrated by a distinct orienting behavior toward a wide range of stimuli. However, in contrast to the PTSD participants, who were mobilized and ready for flight, the resilient participants remained in the preencounter stage where the predator has been identified but is still far away. It can be speculated that resilient participants might control theirsymptoms by permanently scanning the environment for threatening content and by inhibiting the activation of overt reaction.

A cross-sectional design cannot determine the causality of the effects. However, in a tentative analysis, we aimed to explore whether the marked difference between PTSD patients and resilient participants in physiological reactivity to aversive stimuli is constitutional (Guthrie \& Bryant, 2005) or acquired (Orret al., 2003). The regression analysis revealed that vulnerable participants (i.e., individuals with high scores in PTSD symptoms despite relatively few traumatic events) are characterized by an elevated early HR response toward threatening stimuli. Neither depression nor postmigration factors such as the asylum status contributed to the prediction of HR response. Our results give some indication that a pretrauma psychophysiological arousal may represent a vulnerability factor for the development of PTSD, whereas a strong orienting response involving an initial deceleration of HR might represent resilience. However, this hypothesis should be tested in a longitudinal study.

Gender was also a significant predictor for the initial HR response. Yet, because the reanalysis with gender as an additional factor revealed no different outcome, it can be hypothesized that heart rate responses of men and women did not differ substantially. Only if more variables were taken into account did gender receive some predictive value. Results indicate that women show a greater initial heart rate deceleration in response to viewing 
aversive pictures compared to men. This finding is in line with previous studies investigating the differences in heart rate responding toward aversive pictures in men and women (Bradley, Codispoti, Sabatinelli, et al., 2001). Bradley, Codispoti, Sabetelli, et al. (2001) found a stronger deceleration toward aversive pictures in female compared to male subjects and suggested that symbolic picture cues activate the defensive motive system more intensely in women than in men.

What should be noted with respect to the debate about possible gender effects in the present study is that men and women significantly differ with respect to the amount of war and torture they have experienced $(t=3.24, d f=54, p<.005)$. Men reported significantly more types of war and torture events when compared to their female counterparts. This confounding effect was another reason why we decided not to focus our analyses on group differences between men and women. Future studies should ideally include female and male trauma survivors with a comparable quantity and quality of traumatic experience, thereby allowing researchers to clearly disentangle gender effects from effects related to trauma severity.

A limitation of the present study is that group differences cannot be solely attributed to PTSD status because there were substantial differences between groups in terms of comorbid disorders, including depression. However, this problem is inherent in most PTSD research because PTSD is commonly asso- ciated with other disorders. A restriction to participants without comorbid disorders limits the generalization of results to participants with less serious traumatic events who might present with a qualitatively different picture. In addition, the groups differed with respect to medication. Although there is no indication that differential HR reactivity in general is affected by antidepressant medication, this effect cannot be ruled out as a confounding factor. Further studies should try to find samples without medication or increase sample size to allow a subgroup analysis depending on medication status. Finally, to address the question of whether possible avoidance mechanisms might have contributed to the finding of a weaker orienting response in PTSD, future studies should ideally measure eye movements in addition to the HR response.

Our results suggest that trauma-exposed participants with and without PTSD as well as healthy participants can be discriminated on the basis of their pattern of heart rate reactions to emotional stimuli. Moreover, the findings indicate that dysfunctional as well as functional alterations of the defense cascade can be observed in victims of war and torture. The absence of an orienting response to aversive pictures in PTSD patients and the dominance of the freezing state in resilient participants indicate that an orienting response might be adaptive and prevent a maladaptive physiological arousal or flight response after traumatic exposure.

\section{REFERENCES}

Blake, D. D. Weathers, F. W., Nagy, L. M., Kaloupek, D. G., Gusman, F. D., Charney, D. S., et al. (1995). The development of a ClinicianAdministered PTSD Scale. Journal of Traumatic Stress, 8, 75-90.

Blanchard, E. B., Hickling, E. J., Buckley, T. C., Taylor, A. E., Vollmer A., \& Loos, W. R. (1996). Psychophysiology of posttraumatic stress disorder related to motor vehicle accidents: Replication and extension. Journal of Consulting and Clinical Psychology, 64, 742-751

Bracha, H. S. (2004). Freeze, flight, fight, fright, faint: Adaptationis perspectives on the acute stress response spectrum. CNS Spectrums, 9 679-685.

Bradley, M. M., Codispoti, M., Cuthbert, B. N., \& Lang, P. J. (2001) Emotion and motivation I: Defensive and appetitive reactions in picture processing. Emotion, 1, 276-298.

Bradley, M. M., Codispoti, M., Sabatinelli, D., \& Lang, P. J. (2001) Emotion and motivation II: Sex differences in picture processing. Emotion, 1, 300-319.

Bryant, R. A. (2006). Longitudinal psychophysiological studies of heart rate: Mediating effects and implications for treatment. Annols of the New York Academy of Sciences, 1071, 19-26.

Bryant, R. A., \& Harvey, A. G. (1997). Attentional bias in posttraumatic stress disorder. Journal of Traumatic Stress, 10, 635-644.

Buckley, T. C., Blanchard, E. B., \& Neill, W. T. (2000). Information processing and PTSD: A review of the empirical literature. Clinical Psychology Review, 20, 1041-1065.

Buckley, T. C., \& Kaloupek, D. G. (2001). A meta-analytic examination of basal cardiovascular activity in posttraumatic stress disorder. Psychosomatic Medicine, 63, 585-594.

Carson, M. A., Metzger, L. J., Lasko, N. B., Paulus, L. A., Morse, A. E. Pitman, R. K., et al. (2007). Physiologic reactivity to startling tones in female Vietnam nurse veterans with PTSD. Journal of Traumatic Stress, 20, 657-666.

Elbert, T., Rockstroh, B., Kolassa, I., Schauer, M., \& Neuner, F. (2006) The influence of organized violence and terror on brain and mind $-A$ co-constructive perspective. In P. B. Baltes, P. A. Reuter-Lorenz, \& $\mathrm{F}$. Rösler (Eds.), Lifespan development and the brain: The perspective of biocultural co-constructivism (pp. 326-363). Cambridge, UK: Cambridge University Press.

Elsesser, K., Sartory, G., \& Tackenberg, A. (2004). Attention, heart rate and startle response during exposure to trauma-relevant pictures: A comparison of recent trauma victims and patients with posttraumatic stress disorder. Journal of Abnormal Psychology, 113, 289-301.

Fanselow, M. S. (1994). Neural organization of the defensive behavior system responsible for fear. Psychonomic Bulletin and Review, 1, 429438.

Graham, F. K. (1979). Distinguishing among orienting, defense, and startle reflexes. In H. D. Kimmel, E. H. van Olst, \& J. F. Orlebeke (Eds.), The orienting reflex in humans\#\#137-167). Hillsdale, NJ: Erlbaum.

Gray, J. A. (1987). The psychology of fear and stress. Cambridge, UK: Cambridge University Press.

Greenhouse, S., \& Geisser, S. (1959). On methods in the analysis of profile data. Psychometrika, 24, 95-112.

Guthrie, R. M., \& Bryant, R. A. (2005). Auditory startle response in firefighters before and after trauma exposure. American Journal of Psychiatry, 162, 283-290.

Hamm, A. O., Cuthbert, B. N., Globisch, J., \& Vaitl, D. (1997). Fear and the startle reflex: Blink modulation and autonomic response patterns in animal and mutilation fearful subjects. Psychophysiology, 34, 97107

Hodes, R. L., Cook, E. W. 3rd, \& Lang, P. J. (1985). Individual differences in autonomic response: Conditioned association or conditioned fear? Psychophysiology, 22, 545-560.

Lang, P. J., Bradley, M. M., \& Cuthbert, B. N. (1999). International affective picture system (IAPS): Instruction manual and affective ratings. Gainesville, FL: The Center for Research in Psychophysiology, University of Florida.

Lang, P. J., Bradley, M. M., \& Cuthbert, B. N. (2005). International affective struction mamual. Gainesville, FL: University of Florida.

Lang, P. J., Davis, M., \& Ohman, A. (2000). Fear and anxiety: Animal models and human cognitive psychophysiology. Journal of Affective Disorders, 61, 137-159.

Miller, M. W., \& Litz, B. T. (2004). Emotional-processing in posttraumatic stress disorder II: Startle reflex modulation during picture processing. Journal of Abnormal Psychology, 113, 451-463.

Moratti, S., Keil, A., \& Stolarova, M. (2004). Motivated attention in emotional picture processing is reflected by activity modulation in cortical attention networks. NeuroImage, 21, 954-964. 
Neuner, F. Schauer, M., Karunakara, U, Klaschik, C., Robert, C., \& Elbert, T. (2004). Psychological trauma and evidence for enhanced vulnerability for posttraumatic stress disorder through previous trauma among West Nile refugees. BMC Psychiatry, 4, 34

Orr, S. P. Metzger, L. J., Lasko, N. B., Macklin, M. L., Hu, F. B. Shalev, A. Y., et al. (2003). Physiologic responses to sudden, loud tones in monozygotic twins discordant for combat exposure: Association with posttraumatic stress disorder. Archives of General Psychiatry, 60, 283-288.

Orr, S. P., \& Roth, W. T. (2000). Psychophysiological assessment: Clinical applications for PTSD. Journal of Affective Disorders, 61, 225240

Pole, N. (2007). The psychophysiology of posttraumatic stress disorder: A meta-analysis. Psychological Bulletin, 133, 725-746.

Rauch, S. L., Whalen, P. J., Shin, L. M., McInerney, S. C., Macklin, M L., Lasko, N. B., et al. (2000). Exaggerated amygdala response to masked facial stimuli in posttraumatic stress disorder: A functional MRI study. Biological Psychiatry, 47, 769-776.

Rief, W., Hiller, W., \& Heuser, J. (1997). Screening für Somatoforme Störungen (SOMS). Bern: Huber.

Shalev, A. Y., Sahar, T., Freedman, S., Peri, T., Glick, N., Brandes, D., et al. (1998). A prospective study of heart rate response following trauma and the subsequent development of posttraumatic stress disorder. Archives of General Psychiatry, 55, 553-559.
Sheehan, D. V., Lecrubier, Y., Sheehan, K. H., Amorim, P., Janavs, J., Weiller, E., et al. (1998). The Mini-International Neuropsychiatric Interview (M.I.N.I.): The development and validation of a s tructured diagnostic psychiatric interview for DSM-IV and ICD-10. Journal of Clinical Psychiatry, 59(Suppl 20), 22-33; quiz 34-57.

Silove, D., Steel, Z., McGorry, P., \& Mohan, P. (1998). Trauma exposure, postmigration stressors, and symptoms of anxiety, depression and post-traumatic stress in Tamil asylum-seekers: Comparison with refugees and immigrants. Acta Psychiatrica Scandinavica, 97, $175-181$.

Sokolov, Y. N. (1963). Perception and the conditioned reflex (S. W. Waydenfeld, Trans.). New York: Macmillan. (Original work published 1958)

Timberlake, W. (1993). Behavior systems and reinforcement: An integrative approach. Journal of the Experimental Analysis of Behavior, $60,105-128$

Vivo. (2006). Vivo checklist of war, detention and torture events. Retrieved November 8, 2009, from: http://www.vivofoundation.net/.

Williams, J. B. (1988). A structured interview guide for the Hamilton Depression Rating Scale. Archives of General Psychiatry, 45, 742747. 